\title{
Upper Bound of Second Hankel Determinant for Certain Subclasses of Analytic Functions
}

\author{
Ming-Sheng Liu, ${ }^{1}$ Jun-Feng $\mathrm{Xu},{ }^{2}$ and Ming Yang ${ }^{1}$ \\ ${ }^{1}$ School of Mathematical Sciences, South China Normal University, Guangzhou, Guangdong 510631, China \\ ${ }^{2}$ Department of Mathematics, Wuyi University, Jiangmen, Guangdong 529020, China
}

Correspondence should be addressed to Ming-Sheng Liu; liumsh@scnu.edu.cn

Received 19 March 2014; Accepted 25 May 2014; Published 5 June 2014

Academic Editor: V. Ravichandran

Copyright (c) 2014 Ming-Sheng Liu et al. This is an open access article distributed under the Creative Commons Attribution License, which permits unrestricted use, distribution, and reproduction in any medium, provided the original work is properly cited.

In this present investigation, we first give a survey of the work done so far in this area of Hankel determinant for univalent functions. Then the upper bounds of the second Hankel determinant $\left|a_{2} a_{4}-a_{3}^{2}\right|$ for functions belonging to the subclasses $S(\alpha, \beta), K(\alpha, \beta)$, $S_{s}^{*}(\alpha, \beta)$, and $K_{s}(\alpha, \beta)$ of analytic functions are studied. Some of the results, presented in this paper, would extend the corresponding results of earlier authors.

\section{Introduction}

Let $\mathscr{A}$ denote the class of functions of the form

$$
f(z)=z+\sum_{k=2}^{\infty} a_{k} z^{k}
$$

which are analytic in the unit disc $\mathbb{U}=\{z:|z|<1\}$, and let $S$ denote the subclass of $\mathscr{A}$ that is univalent in $\mathbb{U}$. Suppose that $f$ and $g$ are analytic functions in $\mathbb{U}$; we say that $f$ is subordinate to $g$, written $f \prec g$, if there exists a Schwarz function $\omega$, which is analytic in $\mathbb{U}$ with $\omega(0)=0$ and $|\omega(z)|<1$ for all $z \in \mathbb{U}$, such that $f(z)=g(\omega(z)), z \in \mathbb{U}$. In particular, if $g$ is univalent in $\mathbb{U}$, then the subordination is equivalent to $f(0)=g(0)$ and $f(\mathbb{U}) \subset g(\mathbb{U})$.

Let $\mathscr{P}$ be the family of all functions $p$ analytic in $\mathbb{U}$ for which $\mathfrak{R}\{p(z)\}>0$ and

$$
p(z)=1+c_{1} z+c_{2} z^{2}+\cdots
$$

for $z \in \mathbb{U}$.

It is well known that the following correspondence between the class $\mathscr{P}$ and the class of Schwarz functions $\omega$ exists [1]:

$$
p \in \mathscr{P} \Longleftrightarrow p=\frac{1+\omega}{1-\omega} .
$$

Let $S^{*}$ denote the starlike subclass of $S$. It is well known that $f \in S^{*}$ if and only if

$$
\mathfrak{R}\left\{\frac{z f^{\prime}(z)}{f(z)}\right\}>0 \quad(z \in \mathbb{U}) .
$$

Let $K$ denote the class of all functions $f \in \mathscr{A}$ that are convex. Further, $f$ is convex if and only if $z f^{\prime}$ is starlike. Also we know that $K \subset S^{*} \subset S$.

In 1959, Sakaguchi [2] introduced the class $S_{s}^{*}$ of functions starlike with respect to symmetric points, consisting of functions $f \in S$ satisfying

$$
\mathfrak{R}\left\{\frac{2 z f^{\prime}(z)}{f(z)-f(-z)}\right\}>0 \quad(z \in \mathbb{U}) .
$$

In 1977, Das and Singh [3] introduced the class $K_{s}$ of functions convex with respect to symmetric points, which consists of functions $f \in S$ satisfying

$$
\Re\left\{\frac{2\left(z f^{\prime}(z)\right)^{\prime}}{(f(z)-f(-z))^{\prime}}\right\}>0 \quad(z \in \mathbb{U}) .
$$

It is evident that $f \in K_{s}$ if and only if $z f^{\prime} \in S_{s}^{*}$.

In 2007, Wang and Jiang [4] introduced the following subclass. 
Definition 1 (see [4]). Suppose that $0 \leq \alpha \leq 1$ and $0<\beta \leq 1$. Let $S(\alpha, \beta)$ denote the class of functions $f$ in $\mathscr{A}$ satisfying the following inequality:

$$
\left|\frac{z f^{\prime}(z)}{f(z)}-1\right|<\beta\left|\frac{\alpha z f^{\prime}(z)}{f(z)}+1\right| \quad(z \in \mathbb{U}) .
$$

From [4], one knows that the above condition is equivalent to

$$
\frac{z f^{\prime}(z)}{f(z)} \prec \frac{1+\beta z}{1-\alpha \beta z} \quad(z \in \mathbb{U})
$$

which implies that

$$
S(\alpha, \beta) \subset S^{*} \subset S \text {. }
$$

If $\alpha=\beta=1$, then the class $S(\alpha, \beta)$ reduces to the class $S^{*}$. In the similar way, one can easily get the following definitions.

Definition 2. Suppose that $0 \leq \alpha \leq 1$ and $0<\beta \leq 1$. Let $K(\alpha, \beta)$ denote the class of functions $f$ in $\mathscr{A}$ satisfying the following inequality:

$$
\left|\frac{\left(z f^{\prime}(z)\right)^{\prime}}{f^{\prime}(z)}-1\right|<\beta\left|\frac{\alpha\left(z f^{\prime}(z)\right)^{\prime}}{f^{\prime}(z)}+1\right| \quad(z \in \mathbb{U}) .
$$

It is evident that the above condition is equivalent to

$$
\frac{\left(z f^{\prime}(z)\right)^{\prime}}{f^{\prime}(z)} \prec \frac{1+\beta z}{1-\alpha \beta z} \quad(z \in \mathbb{U})
$$

which implies that

$$
K(\alpha, \beta) \subset K \subset S
$$

If $\alpha=1$ and $\beta=1$, then the class $K(\alpha, \beta)$ reduces to the class $K$.

Definition 3. Suppose that $0 \leq \alpha \leq 1$ and $0<\beta \leq 1$. Let $S_{s}^{*}(\alpha, \beta)$ denote the class of functions $f$ in $\mathscr{A}$ satisfying the following inequality:

$$
\left|\frac{2 z f^{\prime}(z)}{f(z)-f(-z)}-1\right|<\beta\left|\frac{2 \alpha z f^{\prime}(z)}{f(z)-f(-z)}+1\right| \quad(z \in \mathbb{U}) \text {. }
$$
lent to

From [5], one knows that the above condition is equiva-

$$
\frac{2 z f^{\prime}(z)}{f(z)-f(-z)} \prec \frac{1+\beta z}{1-\alpha \beta z} \quad(z \in \mathbb{U})
$$

The function class $S_{s}^{*}(\alpha, \beta)$ was introduced and investigated by Sudharsan et al. [6]. If $\alpha=1$ and $\beta=1$, then the class $S_{s}^{*}(\alpha, \beta)$ reduces to the class $S_{s}^{*}$.
Definition 4. Suppose that $0 \leq \alpha \leq 1$ and $0<\beta \leq 1$. Let $K_{s}(\alpha, \beta)$ denote the class of functions $f$ in $\mathscr{A}$ satisfying the following inequality:

$$
\left|\frac{2\left(z f^{\prime}(z)\right)^{\prime}}{(f(z)-f(-z))^{\prime}}-1\right|<\beta\left|\frac{2 \alpha\left(z f^{\prime}(z)\right)^{\prime}}{(f(z)-f(-z))^{\prime}}+1\right|
$$

$(z \in \mathbb{U})$.

It is evident that the above condition is equivalent to

$$
\frac{2\left(z f^{\prime}(z)\right)^{\prime}}{(f(z)-f(-z))^{\prime}} \prec \frac{1+\beta z}{1-\alpha \beta z} \quad(z \in \mathbb{U}) .
$$

If $\alpha=1$ and $\beta=1$, then the class $K_{s}(\alpha, \beta)$ reduces to the class $K_{s}$.

In 1966, Pommerenke [7] stated the qth Hankel determinant for $q \geq 1$ and $n \geq 1$ as

$$
H_{q}(n)=\left|\begin{array}{cccc}
a_{n} & a_{n+1} & \cdots & a_{n+q-1} \\
a_{n+1} & a_{n+2} & \cdots & a_{n+q} \\
\cdots & \cdots & \cdots & \cdots \\
a_{n+q-1} & a_{n+q} & \cdots & a_{n+2 q-2}
\end{array}\right|, \quad\left(a_{1}=1\right) .
$$

This Hankel determinant is useful and has also been considered by several authors. The growth rate of Hankel determinant $H_{q}(n)$ as $n \rightarrow \infty$ was investigated, respectively, when $f$ is a member of certain subclass of analytic functions, such as the class of $p$-valent functions $[7,8]$, the class of starlike functions [7], the class of univalent functions [9], the class of close-to-convex functions [10], the class of strong close-to-convex functions [11], a new class $V_{k}$ [12], and a new class $\widetilde{N}_{k}(\eta, \rho, \beta)$ [13]. Similar to the above discussions, we can also refer to $[14,15]$. Ehrenborg [16] studied the Hankel determinant of exponential polynomials. The Hankel transform of an integer sequence was defined and some of its properties were discussed by Layman [17]. Pommerenke [9] proved that the Hankel determinants of univalent function satisfy

$$
\left|H_{q}(n)\right| \leq K n^{-(1 / 2+\beta) q+3 / 2} .
$$

Later, $\left|H_{2}(n)\right| \leq A n^{1 / 2}$ was also proved by Hayman [18]. One can easily observe that the Fekete and Szegö functional is $H_{2}(1)=a_{3}-a_{2}^{2}$. For results related to the functional, see $[19,20]$. Fekete and Szegö further generalized the estimate $\left|a_{3}-\mu a_{2}^{2}\right|$, where $\mu$ is real and $f \in S$. For results related to the functional, see [21, 22]. In 2010, Hayami and Owa [21, 22] also generalized the estimate $\left|a_{n} a_{n+2}-\mu a_{n}^{2}\right|$ for analytic function. Later, in 2012, Krishna and Ramreddy [23] also generalized the estimate $\left|a_{p+1} a_{p+3}-\mu a_{p+2}^{2}\right|$ for $p$-valent analytic function; see also $[24,25]$.

For our discussion in this paper, we consider the second Hankel determinant in the case of $q=2$ and $n=2$, namely,

$$
H_{2}(2)=\left|\begin{array}{ll}
a_{2} & a_{3} \\
a_{3} & a_{4}
\end{array}\right|=a_{2} a_{4}-a_{3}^{2} .
$$


Janteng et al. [26] have considered the functional $\left|H_{2}(2)\right|$ and found a sharp bound, the subclass of $S$ denoted by $R$, defined as $\mathfrak{R}\left\{f^{\prime}(z)\right\}>0$. In their work, they have shown that if $f \in R$, then $\left|H_{2}(2)\right| \leq 4 / 9$. These authors [27, 28] also studied the second Hankel determinant and sharp bound for the classes of starlike and convex functions, close-to-starlike and close-to-convex functions with respect to symmetric points denoted by $S^{*}, K, S_{c}^{*}$, and $K_{c}$ and have shown that $\left|H_{2}(2)\right| \leq 1,\left|H_{2}(2)\right| \leq 1 / 8,\left|H_{2}(2)\right| \leq 1$, and $\left|H_{2}(2)\right| \leq 1 / 9$, respectively.

Singh [29] established the second Hankel determinant and sharp bound for the classes of close-to-starlike and closeto-convex functions with respect to conjugate and symmetric conjugate points denoted by $S_{c}^{*}, S_{s c}^{*}, K_{c}$, and $K_{s c}$ and has shown that $\left|H_{2}(2)\right| \leq 1,\left|H_{2}(2)\right| \leq 1,\left|H_{2}(2)\right| \leq 1 / 8$, and $\left|H_{2}(2)\right| \leq 1 / 9$, respectively.

Mishra and Gochhayat [30] obtained the sharp bound to $\left|H_{2}(2)\right|$ for the functions in the class denoted by $R_{\lambda}(\alpha, \rho)$, $(0 \leq \lambda<1,|\alpha|<\pi / 2,0 \leq \rho \leq 1)$ and defined as $\Re\left\{e^{i \alpha}\left(\Omega_{z}^{\lambda} f(z) / z\right)\right\}>\rho \cos \alpha$, using the fractional differential operator denoted by $\Omega_{z}^{\lambda} f(z)$ and defined by Owa and Srivastava [31]. These authors have shown that if $f \in R_{\lambda}(\alpha, \rho)$, then $\left|H_{2}(2)\right| \leq\left\{\left((1-\rho)^{2}(2-\lambda)^{2}(3-\lambda)^{2} \cos ^{2} \alpha\right) / 9\right\}$.

Mohammed and Darus [32] have obtained a sharp upper bound to $\left|H_{2}(2)\right|$ for the functions in the class denoted by $S_{m}^{\lambda, n}(\alpha, \sigma),(|\alpha|<\pi / 2,0 \leq \sigma<1)$ and defined as $\Re\left\{e^{i \alpha}\left(\Theta_{m}^{\lambda, n} f(z) / z\right)\right\}>\sigma \cos \alpha$. These authors have proved that if $f \in S_{m}^{\lambda, n}(\alpha, \sigma)$, then $\left|H_{2}(2)\right| \leq\left\{\left(4 m^{2}(1-\sigma)^{2}(1+\right.\right.$ $\left.\left.m)^{2} \cos ^{2} \alpha\right) /\left(3^{2 n}(\lambda+1)^{2}(\lambda+2)^{2}\right)\right\}$.

Similar to the above discussions in a new subclass of analytic function with different operators, we can also refer to $[33,34]$. Singh [35] also obtained a sharp upper bound for the functional $\left|H_{2}(2)\right|$ for the function $f \in M(\alpha)$, where

$$
\begin{gathered}
M(\alpha)=\left\{f \in \mathscr{A}: \mathfrak{R}\left[\frac{z f^{\prime}(z)+\alpha z^{2} f^{\prime \prime}(z)}{(1-\alpha) f(z)+\alpha z f^{\prime}(z)}\right]>0,\right. \\
0 \leq \alpha \leq 1, z \in \mathbb{U}\},
\end{gathered}
$$

and showed that if $f \in M(\alpha)$, then $\left|H_{2}(2)\right| \leq 1 /((1+\alpha)(1+$ $3 \alpha)$ ).

Mehrok and Singh [36] have obtained a sharp upper bound to $\left|H_{2}(2)\right|$ for the function in the classes denoted by $M^{\alpha}$ and $C_{s}^{*(\alpha)}$ and defined as, respectively,

$$
\begin{gathered}
M^{\alpha}=\left\{f \in \mathscr{A}: \Re\left[\left(\frac{z f^{\prime}(z)}{f(z)}\right)^{1-\alpha}\left(\frac{\left(z f^{\prime}(z)\right)^{\prime}}{f^{\prime}(z)}\right)^{\alpha}\right]>0,\right. \\
0 \leq \alpha \leq 1, z \in \mathbb{U}\},
\end{gathered}
$$

$$
\begin{aligned}
& C_{s}^{*(\alpha)}=\{f \in \mathscr{A}: \mathfrak{R} {\left[\left(\frac{2 z f^{\prime}(z)}{f(z)-f(-z)}\right)^{1-\alpha}\right.} \\
&\left.\times\left(\frac{2\left(z f^{\prime}(z)\right)^{\prime}}{(f(z)-f(-z))^{\prime}}\right)^{\alpha}\right]>0, \\
&0 \leq \alpha \leq 1, z \in \mathbb{U}\} .
\end{aligned}
$$

In their work, they proved that if $f \in M^{\alpha}$, then

$$
\begin{aligned}
&\left|H_{2}(2)\right| \\
& \leq \frac{1}{(1+2 \alpha)^{2}} \\
& \quad \times\left[\alpha\left(11+36 \alpha+38 \alpha^{2}+12 \alpha^{3}-\alpha^{4}\right)\right. \\
& \quad \times\left((1+3 \alpha)\left(-4+263 \alpha+603 \alpha^{2}+253 \alpha^{3}+37 \alpha^{4}\right)\right. \\
&\left.\left.\quad \quad \times(1+\alpha)^{4}\right)^{-1}+1\right],
\end{aligned}
$$

and if $f \in C_{s}^{*(\alpha)}$, then $\left|H_{2}(2)\right| \leq 1 /(1+2 \alpha)^{2}$.

Shanmugam et al. [37] established the sharp upper bound of the second Hankel determinant for the classes of $S_{\alpha}^{*}$ and $C_{\alpha}$, defined as, respectively,

$$
\begin{gathered}
S_{\alpha}^{*}=\left\{f \in \mathscr{A}: \mathfrak{R}\left[\frac{z f^{\prime}(z)}{f(z)}+\alpha \frac{z^{2} f^{\prime \prime}(z)}{f(z)}\right]>0, z \in \mathbb{U}\right\}, \\
C_{\alpha}=\left\{f \in \mathscr{A}: \mathfrak{R}\left[\frac{\left(z f^{\prime}(z)+\alpha z^{2} f^{\prime \prime}\left(z^{\prime}\right)\right)^{\prime}}{f^{\prime}(z)}\right]>0, z \in \mathbb{U}\right\} .
\end{gathered}
$$

These authors proved that if $f \in S_{\alpha}^{*}$, then $\left|H_{2}(2)\right| \leq 1 /(1+3 \alpha)^{2}$ and if $f \in C_{\alpha}$, then

$$
\left|H_{2}(2)\right| \leq \frac{1}{144}\left|\frac{280 \alpha^{3}+340 \alpha^{2}+138 \alpha+18}{(1+2 \alpha)^{2}(1+3 \alpha)^{2}(1+4 \alpha)}\right| .
$$

Krishna and Ramreddy [38] obtained a sharp upper bound to the nonlinear functional $\left|H_{2}(2)\right|$ for a new subclass of analytic functions $Q(\alpha, \beta, \gamma),(\alpha, \beta>0,0 \leq \gamma<\alpha+\beta \leq 1)$, defined by

$$
Q(\alpha, \beta, \gamma)=\left\{f \in \mathscr{A}: \mathfrak{R}\left[\alpha \frac{f(z)}{z}+\beta f^{\prime}(z)\right] \geq \gamma, z \in \mathbb{U}\right\} .
$$

These authors proved that if $f \in Q(\alpha, \beta, \gamma)$, then $\left|H_{2}(2)\right| \leq$ $\left[4(\alpha+\beta-\gamma)^{2} /(\alpha+3 \beta)^{2}\right]$.

Similar to the above discussions defined as different classes of analytic functions, we can also refer to [39-49]. 
Raza and Malik [50] studied the third Hankel determinant $H_{3}(1)$ of analytic functions related with lemniscate of Bernoulli; see also [51].

Motivated by the above-mentioned results obtained by different authors in this direction, in this present investigation, we determine the upper bounds of the second Hankel determinant $\mathrm{H}_{2}$ (2) for functions belonging to these classes $S(\alpha, \beta), K(\alpha, \beta), S_{s}^{*}(\alpha, \beta)$, and $K_{s}(\alpha, \beta)$.

\section{Preliminary Results}

In order to prove our main results, we need the following lemmas.

Lemma 5 (see [52]). If the function $p \in \mathscr{P}$ is given by the power series (2), then $\left|c_{k}\right| \leq 2(k=1,2, \ldots)$.

Lemma 6 (see $[53,54]$ ). If the function $p \in \mathscr{P}$ is given by the power series (2), then

$$
2 c_{2}=c_{1}^{2}+\left(4-c_{1}^{2}\right) x
$$

for some $x$ with $|x| \leq 1$ and

$$
\begin{aligned}
4 c_{3}= & c_{1}^{3}+2 c_{1}\left(4-c_{1}^{2}\right) x-c_{1}\left(4-c_{1}^{2}\right) x^{2} \\
& +2\left(4-c_{1}^{2}\right)\left(1-|x|^{2}\right) z
\end{aligned}
$$

for some $z$ with $|z| \leq 1$.

\section{Main Results}

Theorem 7. Let $0 \leq \alpha \leq 1$ and $0<\beta \leq 1$. Suppose that the function $f$ given by (1) is in the class $S(\alpha, \beta)$. Then

$$
\left|a_{2} a_{4}-a_{3}^{2}\right| \leq \frac{1}{4} \beta^{2}(1+\alpha)^{2} \text {. }
$$

The result is sharp, with the extremal function

$$
f_{1}(z)= \begin{cases}z\left(1-\alpha \beta z^{2}\right)^{-(1+\alpha) / 2 \alpha}, & 0<\alpha \leq 1, \\ z e^{\beta z^{2} / 2}, & \alpha=0 .\end{cases}
$$

Proof. Since $f \in S(\alpha, \beta)$, it follows from (8) that there exists a Schwarz function $\omega$, which is analytic in $\mathbb{U}$ with $\omega(0)=0$ and $|\omega(z)|<1$ in $\mathbb{U}$, such that

$$
\frac{z f^{\prime}(z)}{f(z)}=\phi(\omega(z)) \quad(z \in \mathbb{U}),
$$

where

$$
\begin{aligned}
\phi(z)= & \frac{1+\beta z}{1-\alpha \beta z}=1+\beta(1+\alpha) z+\alpha \beta^{2}(1+\alpha) z^{2} \\
& +\alpha^{2} \beta^{3}(1+\alpha) z^{3}+\cdots .
\end{aligned}
$$

Define the function $p$ by

$$
p(z)=\frac{1+\omega(z)}{1-\omega(z)}=1+c_{1} z+c_{2} z^{2}+\cdots .
$$

From (3), we get $p \in \mathscr{P}$ and

$$
\begin{aligned}
\omega(z)= & \frac{p(z)-1}{p(z)+1}=\frac{1}{2} c_{1} z+\frac{1}{2}\left(c_{2}-\frac{1}{2} c_{1}^{2}\right) z^{2} \\
& +\frac{1}{2}\left(c_{3}-c_{1} c_{2}+\frac{1}{4} c_{1}^{3}\right) z^{3}+\cdots .
\end{aligned}
$$

In view of (30), (31), and (33), we have

$$
\begin{aligned}
\frac{z f^{\prime}(z)}{f(z)}=\phi(\omega(z)) & \\
=\phi( & \frac{1}{2} c_{1} z+\frac{1}{2}\left(c_{2}-\frac{1}{2} c_{1}^{2}\right) z^{2} \\
& \left.+\frac{1}{2}\left(c_{3}-c_{1} c_{2}+\frac{1}{4} c_{1}^{3}\right) z^{3}+\cdots\right) \\
=1+ & \frac{1}{2} \beta(1+\alpha) c_{1} z \\
+ & {\left[\frac{1}{2} \beta(1+\alpha)\left(c_{2}-\frac{1}{2} c_{1}^{2}\right)+\frac{1}{4} \alpha \beta^{2}(1+\alpha) c_{1}^{2}\right] z^{2} } \\
+ & {\left[\frac{1}{2} \beta(1+\alpha)\left(c_{3}-c_{1} c_{2}+\frac{1}{4} c_{1}^{3}\right)\right.} \\
& +\frac{1}{2} \alpha \beta^{2}(1+\alpha)\left(c_{2}-\frac{1}{2} c_{1}^{2}\right) c_{1} \\
& \left.+\frac{1}{8} \alpha^{2} \beta^{3}(1+\alpha) c_{1}^{3}\right] z^{3}+\cdots .
\end{aligned}
$$

Similarly,

$$
\begin{aligned}
\frac{z f^{\prime}(z)}{f(z)}= & 1+a_{2} z+\left(2 a_{3}-a_{2}^{2}\right) z^{2} \\
& +\left(3 a_{4}-3 a_{2} a_{3}+a_{2}^{2}\right) z^{3}+\cdots .
\end{aligned}
$$

Comparing the coefficients of $z, z^{2}$, and $z^{3}$ in (34) and (35), we obtain

$$
\begin{aligned}
a_{2}= & \frac{1}{2} \beta(1+\alpha) c_{1}, \\
a_{3}= & \frac{1}{8} \beta(1+\alpha)\left[2 c_{2}+(\beta+2 \alpha \beta-1) c_{1}^{2}\right], \\
a_{4}= & \frac{1}{8} \beta(1+\alpha) \\
& \times\left(\frac{1}{3}-\frac{1}{2} \beta-\frac{7}{6} \alpha \beta+\frac{5}{6} \alpha \beta^{2}+\alpha^{2} \beta^{2}+\frac{1}{6} \beta^{2}\right) c_{1}^{3} \\
& -\frac{1}{2} \beta(1+\alpha)\left(\frac{1}{3}-\frac{1}{4} \beta-\frac{7}{12} \alpha \beta\right) c_{1} c_{2}+\frac{1}{6} \beta(1+\alpha) c_{3} .
\end{aligned}
$$


Thus we have

$$
\begin{aligned}
a_{2} a_{4}-a_{3}^{2}= & -\frac{1}{192} \beta^{2}(1+\alpha)^{2} \\
& \times\left[\left(2 \alpha \beta^{2}+2 \alpha \beta+\beta^{2}-1\right) c_{1}^{4}-4(\alpha \beta-1) c_{1}^{2} c_{2}\right. \\
& \left.\quad-16 c_{1} c_{3}+12 c_{2}^{2}\right],
\end{aligned}
$$

$$
\begin{aligned}
\left|a_{2} a_{4}-a_{3}^{2}\right|= & \frac{1}{192} \beta^{2}(1+\alpha)^{2} \\
& \times \mid\left(2 \alpha \beta^{2}+2 \alpha \beta+\beta^{2}-1\right) c_{1}^{4} \\
& \quad-4(\alpha \beta-1) c_{1}^{2} c_{2}-16 c_{1} c_{3}+12 c_{2}^{2} \mid .
\end{aligned}
$$

Since the functions $p(z)$ and $p\left(e^{i \theta} z\right)(\theta \in \mathbb{R})$ are members of the class $\mathscr{P}$ simultaneously, we assume without loss of generality that $c_{1}>0$. For convenience of notation, we take $c_{1}=c(c \in[0,2])$. By substituting the values of $c_{2}$ and $c_{3}$, respectively, from (26) and (27) in (38), we have

$$
\begin{aligned}
\left|a_{2} a_{4}-a_{3}^{2}\right|= & \frac{1}{192} \beta^{2}(1+\alpha)^{2} \\
& \times \mid(2 \alpha+1) \beta^{2} c^{4}-2 \alpha \beta c^{2}\left(4-c^{2}\right) x \\
& +\left(12+c^{2}\right)\left(4-c^{2}\right) x^{2} \\
& -8 c\left(4-c^{2}\right)\left(1-|x|^{2}\right) z \mid .
\end{aligned}
$$

Using the triangle inequality and $|z| \leq 1$, we have

$$
\begin{aligned}
\left|a_{2} a_{4}-a_{3}^{2}\right| \leq & \frac{1}{192} \beta^{2}(1+\alpha)^{2} \\
& \times\left[(2 \alpha+1) \beta^{2} c^{4}+2 \alpha \beta c^{2}\left(4-c^{2}\right)|x|\right. \\
& +\left(12+c^{2}\right)\left(4-c^{2}\right)|x|^{2} \\
& \left.+8 c\left(4-c^{2}\right)\left(1-|x|^{2}\right)\right] \\
= & \frac{1}{192} \beta^{2}(1+\alpha)^{2} \\
& \times\left[8 c\left(4-c^{2}\right)+(2 \alpha+1) \beta^{2} c^{4}\right. \\
& +2 \alpha \beta c^{2}\left(4-c^{2}\right)|x| \\
& \left.+(c-2)(c-6)\left(4-c^{2}\right)|x|^{2}\right] \\
= & F(c, \mu), \quad(s a y),
\end{aligned}
$$

where $\mu=|x| \leq 1$.
We next maximize the function $F(c, \mu)$ on the closed square $[0,2] \times[0,1]$. Differentiating $F(c, \mu)$ in (40) partially with respect to $\mu$, we get

$$
\begin{aligned}
\frac{\partial F(c, \mu)}{\partial \mu}= & \frac{1}{96} \beta^{2}(1+\alpha)^{2} \\
& \times\left[\alpha \beta c^{2}\left(4-c^{2}\right)+(c-2)(c-6)\left(4-c^{2}\right) \mu\right]
\end{aligned}
$$

For $0<\mu<1$ and for any fixed $c$ with $0<c<2$, from (41), we observe that $\partial F(c, \mu) / \partial \mu>0$. Consequently, $F(c, \mu)$ is an increasing function of $\mu$ and hence it cannot have a maximum value at any point in the interior of the closed square $[0,2] \times$ $[0,1]$. Moreover, for fixed $c \in[0,2]$, we have

$$
\max _{0 \leq \mu \leq 1} F(c, \mu)=F(c, 1)=G(c) \quad(\text { say }) .
$$

From the relations (40) and (42), upon simplification, we obtain

$$
\begin{aligned}
G(c)= & F(c, 1)=\frac{1}{192} \beta^{2}(1+\alpha)^{2} \\
& \times\left[(2 \alpha \beta+\beta+1)(\beta-1) c^{4}+8(\alpha \beta-1) c^{2}+48\right] .
\end{aligned}
$$

Next, since

$$
\begin{aligned}
G^{\prime}(c)= & \frac{1}{48} \beta^{2}(1+\alpha)^{2} c \\
& \times\left[(2 \alpha \beta+\beta+1)(\beta-1) c^{2}+4(\alpha \beta-1)\right],
\end{aligned}
$$

we get that $G^{\prime}(c) \leq 0$ for $0<c \leq 2$ and $G(c)$ has real critical point at $c=0$. Therefore, the maximum of $G(c)$ occurs at $c=0$. Thus, the upper bound of $F(c, \mu)$ corresponds to $\mu=1$ and $c=0$. Hence,

$$
\left|a_{2} a_{4}-a_{3}^{2}\right| \leq \frac{1}{4} \beta^{2}(1+\alpha)^{2}
$$

Equality holds for the function

$$
f_{1}(z)= \begin{cases}z\left(1-\alpha \beta z^{2}\right)^{-(1+\alpha) / 2 \alpha}, & 0<\alpha \leq 1, \\ z e^{\beta z^{2} / 2}, & \alpha=0 .\end{cases}
$$


By calculating, we have

$$
\frac{z f_{1}^{\prime}(z)}{f_{1}(z)}=\frac{1+\beta z^{2}}{1-\alpha \beta z^{2}} \prec \frac{1+\beta z}{1-\alpha \beta z}
$$

and $a_{2}=0, a_{3}=(1 / 2) \beta(1+\alpha)$, and $a_{4}=0$. So $f_{1}(z) \in S(\alpha, \beta)$ and equality holds. This shows that the result is sharp, and the proof of Theorem 7 is complete.

Setting $\alpha=\beta=1$ in Theorem 7, we obtain the following result due to Janteng et al. [27].
Corollary 8. If $f(z) \in S^{*}$, then

$$
\left|a_{2} a_{4}-a_{3}^{2}\right| \leq 1
$$

The result is sharp, with the extremal function

$$
f_{2}(z)=\frac{z}{1-z^{2}}
$$

By using the similar method as in the proof of Theorem 7 , one can similarly prove Theorem 9 .

Theorem 9. Let $0 \leq \alpha \leq 1$ and $0<\beta \leq 1$. Suppose that the function $f$ given by $(1)$ is in the class $K(\alpha, \beta)$. Then

$$
\left|a_{2} a_{4}-a_{3}^{2}\right| \leq \begin{cases}\frac{1}{36} \beta^{2}(1+\alpha)^{2}, & 5 \alpha \beta+\beta-2 \leq 0, \\ \frac{1}{576} \beta^{2}(1+\alpha)^{2}\left[\frac{(5 \alpha \beta+\beta-2)^{2}}{2+\beta(5 \alpha+1)-\beta^{2}(1-\alpha)(2 \alpha+1)}+16\right], & 5 \alpha \beta+\beta-2>0 .\end{cases}
$$

The results are sharp, with the extremal function

$$
f_{3}(z)= \begin{cases}\int_{0}^{z}\left(1-\alpha \beta \mu^{2}\right)^{-(1+\alpha) / 2 \alpha} d \mu, & 0<\alpha \leq 1 \\ \int_{0}^{z} e^{\beta \mu^{2} / 2} d \mu, & \alpha=0\end{cases}
$$

for the case $5 \alpha \beta+\beta-2 \leq 0$, and there is no extremal function for the case $5 \alpha \beta+\beta-2>0$.

Setting $\alpha=\beta=1$ in Theorem 9, one obtains the following result due to Janteng et al. [27].

Corollary 10. If $f(z) \in K$, then

$$
\left|a_{2} a_{4}-a_{3}^{2}\right| \leq \frac{1}{8}
$$

The result is sharp.

Theorem 11. Let $0 \leq \alpha \leq 1$ and $0<\beta \leq 1$. Suppose that the function $f$ given by $(1)$ is in the class $S_{s}^{*}(\alpha, \beta)$. Then

$$
\left|a_{2} a_{4}-a_{3}^{2}\right| \leq \frac{1}{4} \beta^{2}(1+\alpha)^{2}
$$

The result is sharp, with the extremal function

$$
f_{4}(z)= \begin{cases}\int_{0}^{z}\left(1-\alpha \beta \mu^{2}\right)^{-(1+\alpha) / 2 \alpha} & \\ \quad \times\left(\frac{1+\beta \mu^{2}}{1-\alpha \beta \mu^{2}}\right) d \mu, & 0<\alpha \leq 1, \\ \int_{0}^{z} e^{\beta \mu^{2} / 2}\left(1+\beta \mu^{2}\right) d \mu, & \alpha=0 .\end{cases}
$$

Proof. Since $f \in S_{s}^{*}(\alpha, \beta)$, it follows from (14) that there exists a Schwarz function $\omega$, which is analytic in $\mathbb{U}$ with $\omega(0)=0$ and $|\omega(z)|<1$ in $\mathbb{U}$, such that

$$
\frac{2 z f^{\prime}(z)}{f(z)-f(-z)}=\phi(\omega(z)) \quad(z \in \mathbb{U})
$$

where $\phi$ was defined by (31).

In view of (31), (33), and (55), we have

$$
\begin{aligned}
& \frac{2 z f^{\prime}(z)}{f(z)-f(-z)} \\
& =\phi(\omega(z)) \\
& =\phi\left(\frac{1}{2} c_{1} z+\frac{1}{2}\left(c_{2}-\frac{c_{1}^{2}}{2}\right) z^{2}\right. \\
& \left.+\frac{1}{2}\left(c_{3}-c_{1} c_{2}+\frac{c_{1}^{3}}{4}\right) z^{3}+\cdots\right) \\
& =1+\frac{1}{2} \beta(1+\alpha) c_{1} z \\
& +\left[\frac{1}{2} \beta(1+\alpha)\left(c_{2}-\frac{1}{2} c_{1}^{2}\right)+\frac{1}{4} \alpha \beta^{2}(1+\alpha) c_{1}^{2}\right] z^{2} \\
& +\left[\frac{1}{2} \beta(1+\alpha)\left(c_{3}-c_{1} c_{2}+\frac{1}{4} c_{1}^{3}\right)\right. \\
& \quad+\frac{1}{2} \alpha \beta^{2}(1+\alpha)\left(c_{2}-\frac{1}{2} c_{1}^{2}\right) c_{1} \\
& \left.+\frac{1}{8} \alpha^{2} \beta^{3}(1+\alpha) c_{1}^{3}\right] z^{3}+\cdots .
\end{aligned}
$$


Similarly,

$$
\frac{2 z f^{\prime}(z)}{f(z)-f(-z)}=2 a_{2} z+2 a_{3} z^{2}+2\left(2 a_{4}-a_{2} a_{3}\right) z^{3}+\cdots
$$

Comparing the coefficients of $z, z^{2}$, and $z^{3}$ in (56) and (57), we obtain

$$
\begin{aligned}
a_{2}= & \frac{1}{4} \beta(1+\alpha) c_{1}, \\
a_{3}= & \frac{1}{4} \beta(1+\alpha)\left[(\alpha \beta-1) c_{1}^{2}+2 c_{2}\right], \\
a_{4}= & \frac{1}{64} \beta(1+\alpha) \\
& \times\left(2-4 \alpha \beta+3 \alpha^{2} \beta^{2}+\alpha \beta^{2}\right) c_{1}^{3} \\
& +\frac{1}{32} \beta(1+\alpha)(5 \alpha \beta+\beta-4) c_{1} c_{2} \\
& +\frac{1}{8} \beta(1+\alpha) c_{3} .
\end{aligned}
$$

Thus we have

$$
\begin{aligned}
a_{2} a_{4}-a_{3}^{2}=-\frac{1}{256} & \beta^{2}(1+\alpha)^{2} \\
& \times\left[\left(\alpha^{2} \beta^{2}-\alpha \beta^{2}-4 \alpha \beta+2\right) c_{1}^{4}\right. \\
& \left.+(6 \alpha \beta-2 \beta-8) c_{1}^{2} c_{2}-8 c_{1} c_{3}+16 c_{2}^{2}\right]
\end{aligned}
$$

$$
\begin{aligned}
\left|a_{2} a_{4}-a_{3}^{2}\right|= & \frac{1}{256} \beta^{2}(1+\alpha)^{2} \\
& \times \mid\left(\alpha^{2} \beta^{2}-\alpha \beta^{2}-4 \alpha \beta+2\right) c_{1}^{4} \\
& +(6 \alpha \beta-2 \beta-8) c_{1}^{2} c_{2}-8 c_{1} c_{3}+16 c_{2}^{2} \mid .
\end{aligned}
$$

Since the functions $p(z)$ and $p\left(e^{i \theta} z\right)(\theta \in \mathbb{R})$ are members of the class $\mathscr{P}$ simultaneously, we assume without loss of generality that $c_{1}>0$. For convenience of notation, we take $c_{1}=c(c \in[0,2])$. By substituting the values of $c_{2}$ and $c_{3}$, respectively, from (26) and (27) in (60), we have

$$
\begin{aligned}
\left|a_{2} a_{4}-a_{3}^{2}\right|= & \frac{1}{256} \beta^{2}(1+\alpha)^{2} \\
& \times \mid\left(\alpha^{2} \beta^{2}-\alpha \beta^{2}-\alpha \beta-\beta\right) c^{4} \\
& +(3 \alpha \beta-\beta+4) c^{2}\left(4-c^{2}\right) x+2\left(4-c^{2}\right) \\
& \quad \times\left(8-c^{2}\right) x^{2}-4 c\left(4-c^{2}\right)\left(1-|x|^{2}\right) z \mid
\end{aligned}
$$

Using the triangle inequality and $|z|<1$, we have

$$
\begin{aligned}
\left|a_{2} a_{4}-a_{3}^{2}\right| \leq & \frac{1}{256} \beta^{2}(1+\alpha)^{2} \\
& \times\left[\left(\beta+\alpha \beta+\alpha \beta^{2}-\alpha^{2} \beta^{2}\right) c^{4}\right. \\
& \quad+(3 \alpha \beta-\beta+4) c^{2}\left(4-c^{2}\right)|x|+2\left(4-c^{2}\right) \\
& \left.\quad \times\left(8-c^{2}\right)|x|^{2}+4 c\left(4-c^{2}\right)\left(1-|x|^{2}\right)\right] \\
= & \frac{1}{256} \beta^{2}(1+\alpha)^{2} \\
& \times\left[\left(\beta+\alpha \beta+\alpha \beta^{2}-\alpha^{2} \beta^{2}\right) c^{4}+4 c\left(4-c^{2}\right)\right. \\
& \quad+(4+3 \alpha \beta-\beta) c^{2}\left(4-c^{2}\right)|x| \\
& \left.\quad+2(2-c)(4+c)\left(4-c^{2}\right)|x|^{2}\right] \\
= & F(c, \mu), \quad(s a y),
\end{aligned}
$$

where $\mu=|x| \leq 1$.

We next maximize the function $F(c, \mu)$ on the closed square $[0,2] \times[0,1]$. Differentiating $F(c, \mu)$ in (62) partially with respect to $\mu$, we get

$$
\begin{aligned}
\frac{\partial F(c, \mu)}{\partial \mu}= & \frac{1}{256} \beta^{2}(1+\alpha)^{2} \\
& \times\left[(4+3 \alpha \beta-\beta) c^{2}\left(4-c^{2}\right)\right. \\
& \left.+4(2-c)(4+c)\left(4-c^{2}\right) \mu\right] .
\end{aligned}
$$

For $0<\mu<1$ and for any fixed $c$ with $0<c<2$, from (63), we observe that $\partial F(c, \mu) / \partial \mu>0$. Consequently, $F(c, \mu)$ is an increasing function of $\mu$ and hence it cannot have a maximum value at any point in the interior of the closed square $[0,2] \times$ $[0,1]$. Moreover, for fixed $c \in[0,2]$, we have

$$
\max _{0 \leq \mu \leq 1} F(c, \mu)=F(c, 1)=G(c) \quad \text { (say). }
$$

From the relations (62) and (64), upon simplification, we obtain

$$
\begin{aligned}
G(c)= & F(c, 1) \\
= & \frac{1}{256} \beta^{2}(1+\alpha)^{2} \\
& \times\left[\left(2 \beta-2 \alpha \beta+\alpha \beta^{2}-\alpha^{2} \beta^{2}-2\right) c^{4}\right. \\
& \left.\quad+4(3 \alpha \beta-\beta-2) c^{2}+64\right] .
\end{aligned}
$$

Next, since

$$
\begin{aligned}
G^{\prime}(c)= & \frac{1}{64} \beta^{2}(1+\alpha)^{2} c \\
& \times\left[\left(2 \beta-2 \alpha \beta+\alpha \beta^{2}-\alpha^{2} \beta^{2}-2\right) c^{2}\right. \\
& +2(3 \alpha \beta-\beta-2)],
\end{aligned}
$$


we get that $G^{\prime}(c) \leq 0$ for $0<c \leq 2$ and $G(c)$ has real critical point at $c=0$. Therefore, the maximum of $G(c)$ occurs at $c=0$. Thus, the upper bound of $F(c, \mu)$ corresponds to $\mu=1$ and $c=0$. Hence,

$$
\left|a_{2} a_{4}-a_{3}^{2}\right| \leq \frac{1}{4} \beta^{2}(1+\alpha)^{2} .
$$

Equality holds for the function

$$
f_{4}(z)= \begin{cases}\int_{0}^{z}\left(1-\alpha \beta \mu^{2}\right)^{-(1+\alpha) / 2 \alpha} \\ \quad \times\left(\frac{1+\beta \mu^{2}}{1-\alpha \beta \mu^{2}}\right) d \mu, & 0<\alpha \leq 1, \\ \int_{0}^{z} e^{\beta \mu^{2} / 2}\left(1+\beta \mu^{2}\right) d \mu, & \alpha=0 .\end{cases}
$$

By calculating, we have

$$
\frac{2 z f_{4}^{\prime}(z)}{f_{4}(z)-f_{4}(-z)}=\frac{1+\beta z^{2}}{1-\alpha \beta z^{2}} \prec \frac{1+\beta z}{1-\alpha \beta z}
$$

and $a_{2}=0, a_{3}=-(1 / 2) \beta(1+\alpha)$, and $a_{4}=0$. So $f_{4}(z) \in S(\alpha, \beta)$ and equality holds. This shows that the result is sharp, and the proof of Theorem 11 is complete.

Setting $\alpha=\beta=1$ in Theorem 11, we obtain the following result due to Janteng et al. [28].

Corollary 12. If $f(z) \in S_{s}^{*}$, then

$$
\left|a_{2} a_{4}-a_{3}^{2}\right| \leq 1
$$

The result is sharp, with the extremal function

$$
f_{5}(z)=\int_{0}^{z} \frac{1+\mu^{2}}{\left(1-\mu^{2}\right)^{2}} d \mu .
$$

By using the similar method as in the proof of Theorem 11, one can similarly prove Theorem 13 .

Theorem 13. Let $0 \leq \alpha \leq 1$ and $0<\beta \leq 1$. Suppose that the function $f(z)$ given by $(1)$ is in the class $K_{s}(\alpha, \beta)$. Then

$$
\left|a_{2} a_{4}-a_{3}^{2}\right| \leq \frac{1}{36} \beta^{2}(1+\alpha)^{2} .
$$

The result is sharp, with the extremal function

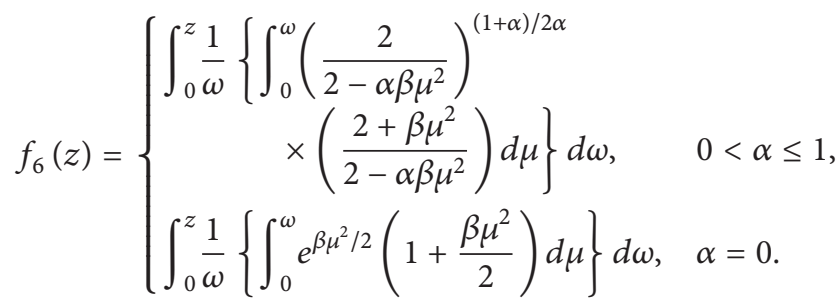

Setting $\alpha=\beta=1$ in Theorem 13, one obtains the following result due to Janteng et al. [28].
Corollary 14. If $f(z) \in K_{s}$, then

$$
\left|a_{2} a_{4}-a_{3}^{2}\right| \leq \frac{1}{9}
$$

The result is sharp, with the extremal function

$$
f_{7}(z)=2 \int_{0}^{z} \frac{1}{\omega}\left\{\int_{0}^{\omega} \frac{2+\mu^{2}}{\left(2-\mu^{2}\right)^{2}} d \mu\right\} d \omega .
$$

\section{Conflict of Interests}

The authors declare that they have no conflict of interests regarding the publication of this paper.

\section{Acknowledgments}

The work was financially supported by Foundation for Distinguished Young Talents in Higher Education of Guangdong China (no. 2013LYM0093) and Training plan for the Outstanding Young Teachers in Higher Education of Guangdong (no. Yq 2013159). The authors are also grateful to the anonymous referees and Professor V. Ravichandran for making many valuable suggestions that improved the quality and the readability of this paper.

\section{References}

[1] I. Graham and G. Kohr, Geometric Function Theory in One and Higher Dimensions, Marcel Dekker, New York, NY, USA, 2003.

[2] K. Sakaguchi, "On a certain univalent mapping," Journal of the Mathematical Society of Japan, vol. 11, pp. 72-75, 1959.

[3] R. N. Das and P. Singh, "On subclasses of schlicht mapping," Indian Journal of Pure and Applied Mathematics, vol. 8, no. 8, pp. 864-872, 1977.

[4] Z. G. Wang and Y. P. Jiang, "On certain subclasses of closeto-convex and quasi-convex functions with respect to $2 k$ symmetric conjugate points," Journal of Mathematics and Applications, vol. 29, pp. 167-179, 2007.

[5] Z. G. Wang, C. Y. Gao, M. S. Liu, and M. X. Liao, "On subclasses of close-to-convex and quasi-convex functions with respect to k-symmetric points," Advances in Mathematics, vol. 38, no. 1, pp. 44-56, 2009.

[6] T. V. Sudharsan, P. Balasubrahmanyam, and K. G. Subramanian, "On functions starlike with respect to symmetric and conjugate points," Taiwanese Journal of Mathematics, vol. 2, no. 1, pp. 57$68,1998$.

[7] C. Pommerenke, "On the coefficients and Hankel determinants of univalent functions," Journal of the London Mathematical Society, vol. 41, pp. 111-122, 1966.

[8] J. W. Noonan and D. K. Thomas, "On the Hankel determinants of areally mean $p$-valent functions," Proceedings of the London Mathematical Society, vol. 25, pp. 503-524, 1972.

[9] C. Pommerenke, "On the Hankel determinants of univalent functions," Mathematika, vol. 14, pp. 108-112, 1967.

[10] K. I. Noor, "On the Hankel determinants of close-to-convex univalent functions," International Journal of Mathematics and Mathematical Sciences, vol. 3, no. 3, pp. 447-481, 1980.

[11] K. I. Noor, "On certain analytic functions related with strongly close-to-convex functions," Applied Mathematics and Computation, vol. 197, no. 1, pp. 149-157, 2008. 
[12] K. I. Noor, "Hankel determinant problem for the class of functions with bounded boundary rotation," Revue Roumaine de Mathématiques Pures et Appliquées, vol. 28, no. 8, pp. 731739, 1983.

[13] W. UI-Haq and K. I. Noor, "A certain class of analytic functions and the growth rate of Hankel feterminant," Journal of Inequalities and Applications, vol. 2012, article 309, 2012.

[14] M. Arif, K. I. Noor, and M. Raza, "Hankel determinant problem of a subclass of analytic functions," Journal of Inequalities and Applications, vol. 2012, pp. 22-27, 2012.

[15] M. Arif, K. I. Noor, M. Raza, and W. Haq, "Some properties of a generalized class of analytic functions related with Janowski functions," Abstract and Applied Analysis, vol. 2012, Article ID 279843, 11 pages, 2012.

[16] R. Ehrenborg, "The Hankel determinant of exponential polynomials," The American Mathematical Monthly, vol. 107, no. 6, pp. 557-560, 2000.

[17] J. W. Layman, "The Hankel transform and some of its properties," Journal of Integer Sequences, vol. 4, no. 1, pp. 1-11, 2001.

[18] W. K. Hayman, "On the second Hankel determinant of mean univalent functions," Proceedings of the London Mathematical Society, vol. 18, no. 3, pp. 77-94, 1968.

[19] R. M. Ali, S. K. Lee, V. Ravichandran, and S. Supramaniam, "The Fekete-Szegö coefficient functional for transforms of analytic functions," Bulletin of the Iranian Mathematical Society, vol. 35, no. 2, pp. 119-142, 2009.

[20] R. M. Ali, V. Ravichandran, and S. Supramaniam, "Coefficient bounds for $p$-valent functions," Applied Mathematics and Computation, vol. 187, no. 1, pp. 35-46, 2007.

[21] T. Hayami and S. Owa, "Coefficient estimates for analytic functions concerned with Hankel determinant," Fractional Calculus \& Applied Analysis, vol. 13, no. 4, pp. 367-384, 2010.

[22] T. Hayami and S. Owa, "Generalized Hankel determinant for certain classes," International Journal of Mathematical Analysis, vol. 4, no. 52, pp. 2573-2585, 2010.

[23] D. V. Krishna and T. Ramreddy, "Hankel determinant for $p$ valent starlike and convex functions of order $\alpha$," Novi Sad Journal of Mathematics, vol. 42, no. 2, pp. 89-102, 2012.

[24] T. Hayami and S. Owa, "Hankel determinant for $p$-valently starlike and convex functions of order $\alpha$," General Mathematics, vol. 17, no. 4, pp. 29-44, 2009.

[25] T. Hayami and S. Owa, "Applications of Hankel determinant for $p$-valently starlike and convex functions of order $\alpha$," Far East Journal of Applied Mathematics, vol. 46, no. 1, pp. 1-23, 2010.

[26] A. Janteng, S. A. Halim, and M. Darus, "Coefficient inequality for a function whose derivative has a positive real part," Journal of Inequalities in Pure and Applied Mathematics, vol. 7, no. 2, pp. $1-5,2006$.

[27] A. Janteng, S. A. Halim, and M. Darus, "Hankel determinant for starlike and convex functions," International Journal of Mathematical Analysis, vol. 1, no. 13, pp. 619-625, 2007.

[28] A. Janteng, S. A. Halim, and M. Darus, "Hankel determinant for functions starlike and convex with respect to symmetric points," Journal of Quality Measurement and Analysis, vol. 2, no. 1, pp. 37-43, 2006.

[29] G. Singh, "Hankel determinant for analytic functions with respect to other points," Engineering Mathematics Letters, vol. 2, no. 2, pp. 115-123, 2013.

[30] A. K. Mishra and P. Gochhayat, "Second Hankel determinant for a class of analytic functions defined by fractional derivative," International Journal of Mathematics and Mathematical Sciences, vol. 2008, Article ID 153280, 10 pages, 2008.
[31] S. Owa and H. M. Srivastava, "Univalent and starlike generalized hypergeometric functions," Canadian Journal of Mathematics, vol. 39, no. 5, pp. 1057-1077, 1987.

[32] A. Mohammed and M. Darus, "Second Hankel determinant for a class of analytic functions defined by a linear operator," Tamkang Journal of Mathematics, vol. 43, no. 3, pp. 455-462, 2012.

[33] W. R. Ibrahim, "Bounded nonlinear functional derived by the generalized Srivastava-Owa fractional differential operator," International Journal of Analysis, vol. 2013, Article ID 542828, 7 pages, 2013.

[34] A. K. Mishra and S. N. Kund, "The second Hankel determinant for a class of analytic functions associated with the CarlsonShaffer operator," Tamkang Journal of Mathematics, vol. 44, no. 1, pp. 73-82, 2013.

[35] G. Singh, "Hankel determinant for a new subclass of analytic functions," Scientia Magna, vol. 8, no. 4, pp. 61-65, 2012.

[36] B. S. Mehrok and G. Singh, "Estimate of second Hankel determinant for certain classes of analytic functions," Scientia Magna, vol. 8, no. 3, pp. 85-94, 2012.

[37] G. Shanmugam, B. Adolf Stephen, and K. G. Subramanian, "Second Hankel determinant for certain classes of analytic functions," Bonfring International Journal of Data Mining, vol. 2, no. 2, pp. 57-60, 2012.

[38] D. V. Krishna and T. Ramreddy, "Coefficient inequality for certain subclass of analytic functions," Armenian Journal of Mathematics, vol. 4, no. 2, pp. 98-105, 2012.

[39] A. Abubaker and M. Darus, "Hankel determinant for a class of analytic functions involving a generalized linear differential operator," International Journal of Pure and Applied Mathematics, vol. 69, no. 4, pp. 429-435, 2011.

[40] M. H. Al-Abbadi and M. Darus, "Hankel determinant for certain class of analytic function defined by geberalized derivative operator," Tamkang Journal of Mathematics, vol. 43, no. 3, pp. 445-453, 2012.

[41] D. Bansal, "Upper bound of second Hankel determinant for a new class of analytic functions," Applied Mathematics Letters, vol. 26, no. 1, pp. 103-107, 2013.

[42] V. K. Deekonda and R. Thoutreddy, "An upper bound to the second Hankel determinant for a subclass of analytic functions," Bulletin of International Mathematical Virtual Institute, vol. 4, no. 1, pp. 17-26, 2014.

[43] D. V. Krishna and T. Ramreddy, "Coefficient inequality for certain subclasses of analytic functions," New Zealand Journal of Mathematics, vol. 42, pp. 217-228, 2012.

[44] S. K. Lee, V. Ravichandran, and S. Supramaniam, "Bounds for the second Hankel determinant of certain univalent functions," Journal of Inequalities and Applications, vol. 2013, article 281, 17 pages, 2013.

[45] G. Murugusundaramoorthy and N. Magesh, "Coefficient inequalities for certain classes of analytic functions associated with Hankel determinant," Bulletin of Mathematical Analysis and Applications, vol. 1, no. 3, pp. 85-89, 2009.

[46] N. Mohamed, D. Mohamad, and S. C. Soh, "Second Hankel determinant for certain generalized classes of analytic functions," International Journal of Mathematical Analysis, vol. 6, no. 17, pp. 807-812, 2012.

[47] J. W. Noonan and D. K. Thomas, "On the second Hankel determinant of areally mean $p$-valent functions," Transactions of the American Mathematical Society, vol. 223, no. 2, pp. 337346, 1976. 
[48] S. Verma, S. Gupta, and S. Singh, "Bounds of Hankel determinant for a class of univalent functions," International Journal of Mathematics and Mathematical Sciences, vol. 2012, Article ID 147842, 6 pages, 2012.

[49] A. Yahya, S. C. Soh, and D. Mohamad, "Second Hankel determinant for a class of a generalised Sakaguchi class of analytic functions," International Journal of Mathematical Analysis, vol. 7, no. 33, pp. 1601-1608, 2013.

[50] M. Raza and S. N. Malik, "Upper bound of third Hankel determinant for a class of analytic functions related with lemniscate of Bernoulli," Journal of Inequalities and Applications, vol. 2013, article 412, 2013.

[51] K. O. Babalola, "On $H_{3}(1)$ Hankel determinants for some classes of univalent functions," in Inequality Theory and Applications, vol. 6, pp. 1-7, Nova Science, 2010.

[52] P. L. Duren, "Univalent functions," in Grundlehren der Mathematischen Wissenschaften, vol. 259, Springer, New York, NY, USA, 1983.

[53] R. J. Libera and E. J. Złotkiewicz, "Early coefficients of the inverse of a regular convex function," Proceedings of the American Mathematical Society, vol. 85, no. 2, pp. 225-230, 1982.

[54] R. J. Libera and E. J. Złotkiewicz, "Coefficient bounds for the inverse of a function with derivative in $\mathscr{P}$," Proceedings of the American Mathematical Society, vol. 87, no. 2, pp. 251-257, 1983. 


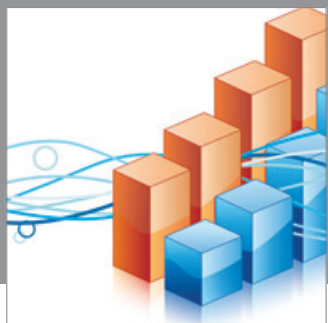

Advances in

Operations Research

mansans

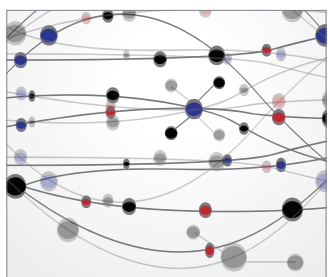

The Scientific World Journal
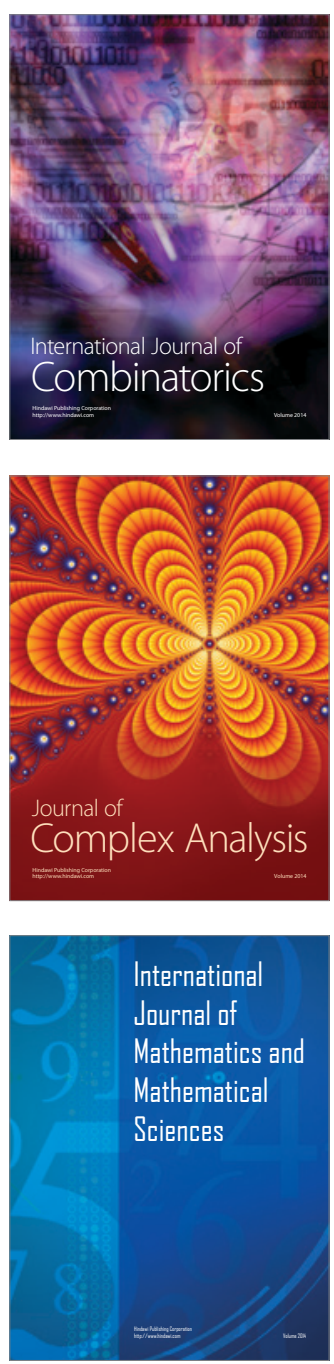
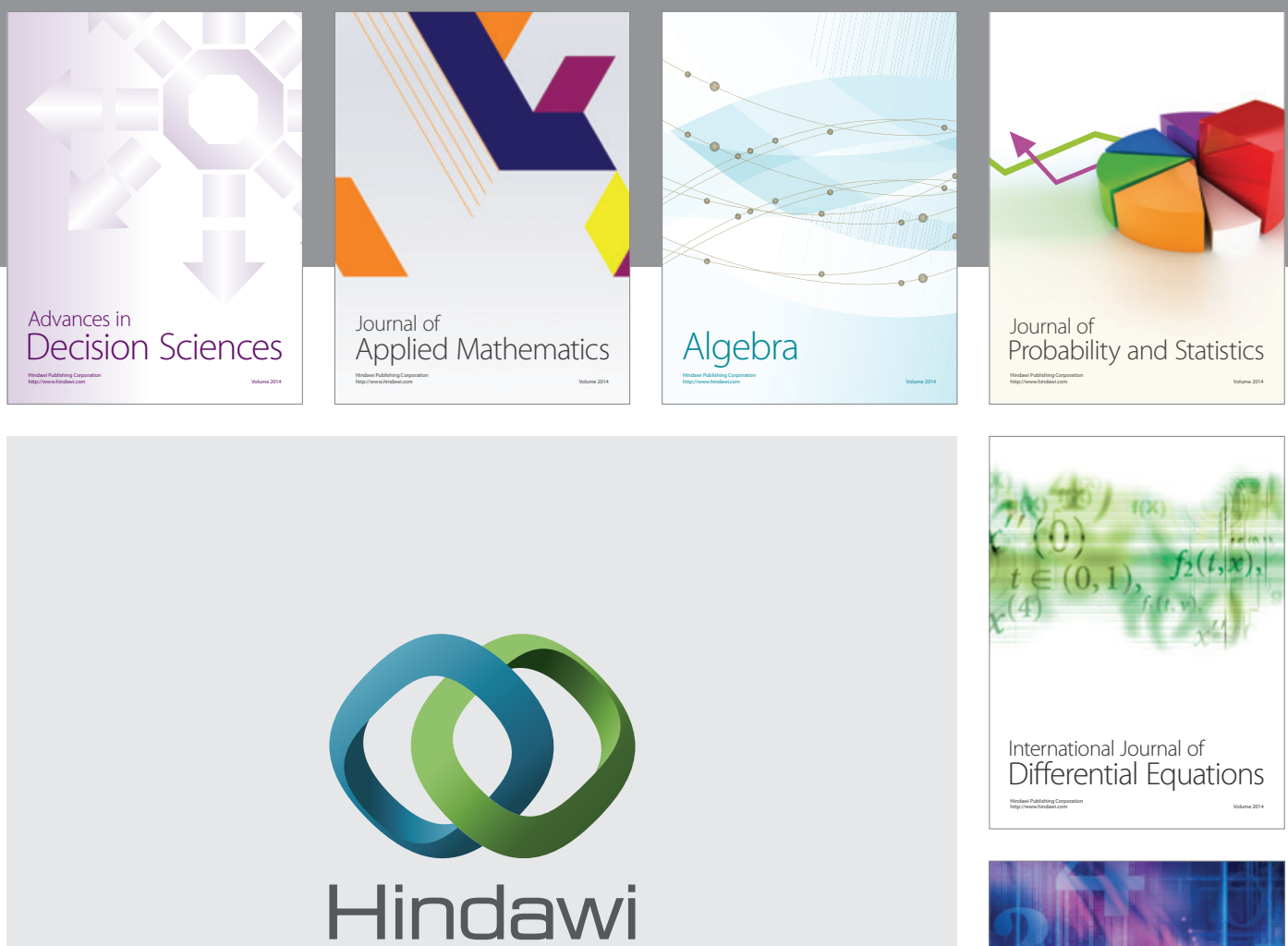

Submit your manuscripts at http://www.hindawi.com
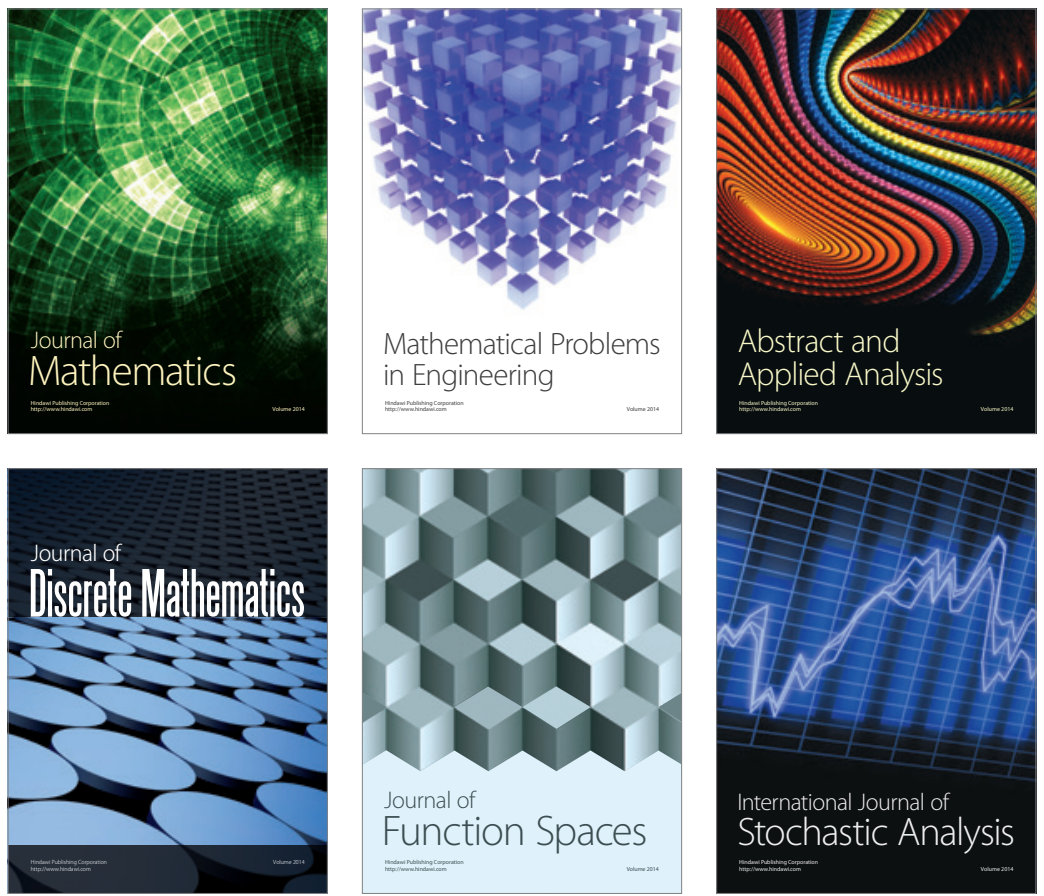

Journal of

Function Spaces

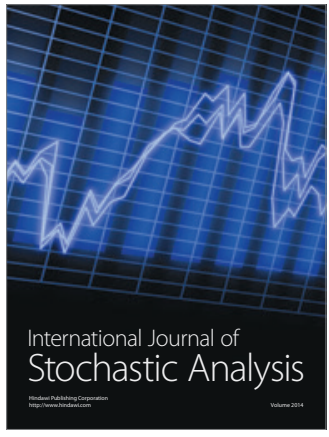

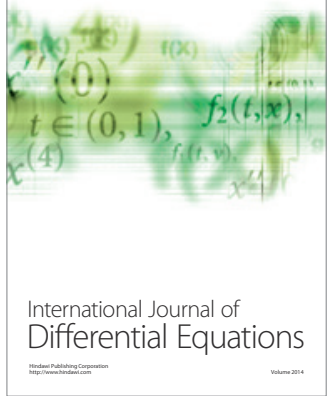
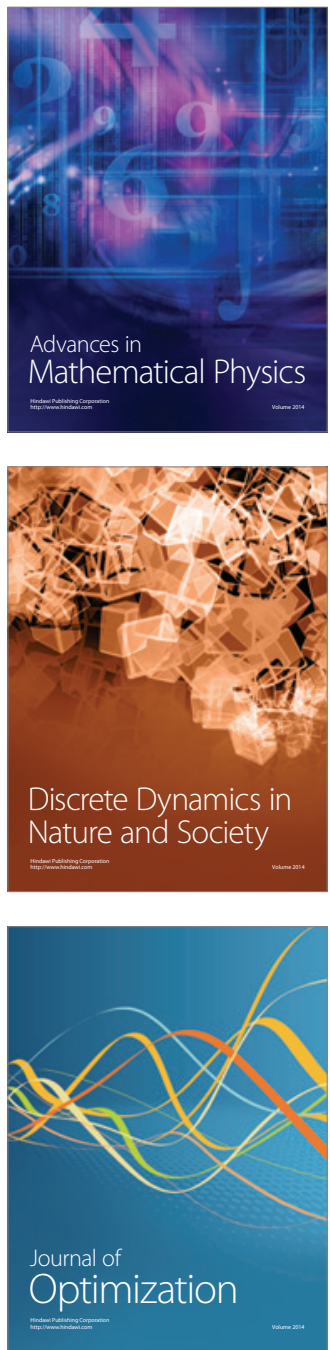